\title{
Performance Analysis of Filters for Speckle Reduction in Medical Ultrasound Images
}

\author{
R.Vanithamani \\ Department of Biomedical \\ Instrumentation Engineering, \\ Faculty of Engineering, \\ Avinashilingam University for Women, \\ Coimbatore, Tamilnadu, India.
}

\author{
G.Umamaheswari \\ Department of Electronics and \\ Communication Engineering, \\ PSG College of Technology, \\ Coimbatore, Tamilnadu, India.
}

\begin{abstract}
Speckle is a random multiplicative noise which obscures the perception and extraction of fine details in ultrasound image and despeckling is necessary to improve the visual quality for better diagnoses. Preliminary treatment of images before segmentation and classification includes despeckling as one of the important steps. This paper aims at introducing the possible range of image speckle corrections available. The performances of different filters - Mean, Lee, Kuan, Frost, Median, Homomorphic, Speckle Reducing Anisotropic Diffusion(SRAD), Non Linear Coherent Diffusion(NCD) are compared on the basis of Peak Signal to Noise Ratio(PSNR), Signal to Noise Ratio(SNR), Root Mean Square Error(RMSE), Structure Similarity Index(SSIM), Image Quality Index(IMGQ) and Edge Preservation Factor(EPF).
\end{abstract}

\section{General Terms}

Digital Image Processing

\section{Keywords}

Speckle noise, ultrasound image, despeckling, anisotropic diffusion, despeckling filters.

\section{INTRODUCTION}

Ultrasound imaging system is an important imaging modality in medical diagnoses. Features like noninvasive nature, low cost, portability, and real-time image formation capacity are making this diagnostic tool attractive. One of the major drawbacks of ultrasound images is the poor image quality due to speckle noise [3]. Ultrasound images are very difficult to diagnose because of the existence of speckle which hampers the perception and the extraction of fine details from the image. Only skilled radiologists can make an effective diagnosis and hence limiting its use in a wide medical network. Ultrasonic speckle is an interference effect caused by the scattering of the ultrasonic beam from microscopic tissues inhomogeneities [1]. This is comparable to the phenomena encountered in laser, microwave and radar imaging. The resulting granular pattern does not correspond to the actual tissue microstructure but it is the speckle pattern which tends to mask the presence of low-contrast lesions. Subsequently, this reduces the ability of a human observer to resolve fine details [12]. Hence, speckle suppression by means of digital image processing is one of the techniques to improve the image quality and possibly the diagnostic potential of medical ultrasound imaging.

\section{MODEL OF SPECKLE NOISE}

The speckle noise model may be approximated as multiplicative [6] and is given by

$$
I i, j=R i, j \quad u i, j+\alpha i, j
$$

Where $I i, j$ is the noisy image and $R i, j$ denotes the intensity of the image without speckle, $\mathrm{u}(\mathrm{t})$ and $\alpha(\mathrm{t})$ are the multiplicative and additive components of the speckle noise respectively. When applied to ultrasound images, only the multiplicative component $u$ of the noise is considered, hence, (1) can be considerably simplified by disregarding the additive noise term. This leads to the following simplified model (2):

$$
I t=R t u t
$$

Where $t=(i, j)$ is the spatial coordinates of the current pixel. Based on this simple model many have proposed various speckle filtering methods and the most efficient filter methods are discussed in this paper.

\section{DESPECKLING FILTERS}

The spatial domain filters for despeckling of ultrasound images can be categorized as linear and non linear filters

\subsection{Linear filters}

\subsubsection{Mean filter}

The mean filter also called averaging filter [5] replaces the value of every pixel in an image by the average of the gray levels in the neighborhood. It has the effect of smoothing and blurring the image and it is optimal filter for Gaussian noise. Since the speckle noise is multiplicative, the simple mean filter is not effective.

\subsubsection{Adaptive mean filter}

The adaptive mean filters have been proposed in order to reduce blurring in the smoothing process. They adapt to the properties of the image locally and remove speckles from the image. The local image statistics such as mean, variance and spatial correlation are used by these filters to effectively detect, preserve edges and 
features. The standard adaptive mean filters for speckle reductions are Lee[8], Kuan[7] and Frost[4]. They are based on the multiplicative model as in (2).The Lee and kuan filter has the general form

$$
\hat{R}(t)=\bar{I}(t)+[I(t)-\bar{I}(t)] \times W(t)
$$

$\mathrm{W}$ is the weighting function ranging between 0 for flat regions and 1 for regions with high signal activity, $\bar{I}$ is the average of pixels in a moving window and $R(i, j)$ is the output of the filter. The weighting function for the Lee filter is calculated according to (4).

$$
W(t)=1-\frac{C_{u}^{2}}{C_{I}^{2}(t)}
$$

Where $C_{u}=\frac{\sigma_{u}}{\bar{u}}$ and $C_{I}=\frac{\sigma_{I}}{\bar{I}}$ are the coefficients of variations of the noise $u$ and the image $I$.

The weighting function of the kuan filter is defined as

$$
W(t)=\frac{1-C_{u}^{2} / C_{I}^{2}(t)}{1+C_{u}^{2}}
$$

From the equations the difference between the two filters is only the term $1+C_{u}^{2}$.In the homogeneous regions $C_{u}{ }^{2}=C_{I}{ }^{2}$ and the value of $\mathrm{W}$ approaches 0 , which makes the filter to act like a mean filter. . In the areas of high variance like edges, $C_{I}^{2}=\infty$, and the value of $\mathrm{W}$ approaches 1 , which tends to preserve the originally observed image, which makes the filters to act like an all pass filter.

The Frost filter can be represented as

$$
\hat{R}(i, j)=\sum_{r} \sum_{s} m(i+r, j+s) \times I(i+r, j+s)
$$

Where $\mathrm{r}$ and $\mathrm{s}$ are the indices of the filter window and $\mathrm{m}$ is the weighting function.

$$
m(i+r, j+s)=K_{0} \exp \left[-K C_{I}^{2}(t) \sqrt{r^{2}+s^{2}}\right]
$$

Where $\mathrm{K}_{0}$ is a normalizing constant and $\mathrm{K}$ is a damping factor. The damping factor $\mathrm{K}$ is chosen such that in homogeneous areas $K C_{I}{ }^{2}$ approaches 0 and the value of $\mathrm{m}$ approaches 1 , which makes filter act like a mean filter. In the areas where edges exists $K C_{I}{ }^{2}$ becomes so large that the value of $m$ approaches 0 for the pixels surrounding $(i, j)$, and remain 1 for the pixel $(i, j)$.This makes the filter to preserve the originally observed image. The classical Lee, kuan and Frost filters are only reliable in a bounded field. In enhanced Lee and kuan filters the image is divided into three classes based on coefficient of variation $C_{I}(t)$.If $C_{I}(t)$ is below a lower threshold, pure averaging is used and when it is above threshold all pass filter is performed. When it is between the two thresholds, standard lee and Frost filters are applied. The enhanced filters adequately average the homogeneous areas and preserve the edges better than the standard filters.

\subsection{Non linear filters}

\subsubsection{Median filter}

Median filtering [9] is a nonlinear filtering method, which is used to remove the 'speckle' noise from an Ultrasound image. It replaces the original gray level of a pixel by the median of gray values of pixels in a specific neighborhood. This filter is popular for reducing the noise without blurring the edges of the image. The median filter is also called the order specific filter because it is based on statistics derived from ordering the elements of a set rather than taking the mean.

\subsubsection{Homomorphic filter}

Homomorphic filter [13] is a generalized technique involving a nonlinear mapping of an image to a different domain in which linear filter techniques are applied, followed by mapping back to the original domain for the purpose of image enhancement and restoration. It simultaneously normalizes the brightness across an image and increases the contrast. Most importantly, homomorphic filter despeckling methods take the advantage of logarithmic transformation, which converts multiplicative noise to additive noise. High boost butter worth filter is used here to reject the resulting additive noise.

\subsubsection{Diffusion filter}

In the case of diffusion filtering the direction and strength of the diffusion are controlled by an edge detection function. It removes speckles and at the same time enhances the edges. It removes speckles by modifying the image via solving a Partial Differential Equation (PDE). Perona and malik proposed the nonlinear PDE for smoothing image in continuous domain.

$$
\left\{\begin{array}{l}
\frac{\partial I}{\partial t}=\partial i v[c(|\nabla I|) . \nabla I] \\
I(t=0)=I_{0}
\end{array}\right.
$$

Where $\nabla$ is the gradient operator, $\partial i v$ is the divergence operator, || denotes the magnitude, $c(|\nabla I|)$ is the diffusion coefficient and $I_{0}$ is the original image. The diffusion coefficient function $c(|\nabla I|)$ should monotonically decrease, the diffusion decreases as the gradient strength increases, and the diffusion is stopped across edges. As the anisotropic diffusion performs well with additive Gaussian noise, Speckle Reducing Anisotropic Diffusion (SRAD)[16] is proposed for speckled images without logarithmic compression. Just as lee and frost filters utilize the coefficient of variation in adaptive filtering, SRAD exploits the instantaneous coefficient of variation, which serves as edge detector in speckled images. The function exhibits high values at edges and produces low values in homogeneous regions. Thus it ensures the mean 
preserving behavior in the homogeneous regions and edge preserving and edge enhancing at the edges. Nonlinear coherent enhancement diffusion (NCD) is another method for handling speckle noise. Unlike SRAD, NCD works with the US images after logarithmic compression.

\section{IMAGE QUALITY EVALUATION METRICS}

The performance of each filter is evaluated quantitatively for Liver ultrasound image with speckle noise using the quality metrics like Root Mean Square Error(RMSE), Signal-to - Noise Ratio(SNR),Peak Signal-to - Noise Ratio(PSNR),Edge Preservation Factor(EPF), Structure Similarity Index(SSIM) and Image Quality Index(IMGQ).Let $x$ and y denote the original and denoised image.

SNR: Signal to Noise Ratio (SNR) [10] compares the level of desired signal to the level of background noise. The higher the ratio, the less obtrusive the background noise is.

$$
\mathrm{SNR}=10 . \log _{10} \frac{\sum_{i=1}^{M} \sum_{j=1}^{N}\left(x_{i, j}^{2}+y_{i, j}^{2}\right)}{\sum_{i=1}^{M} \sum_{j=1}^{N}\left(x_{i, j}-y_{i, j}\right)^{2}}
$$

Where $\mathrm{M}$ and $\mathrm{N}$ are the width and height of the image. The larger SNR values correspond to good quality image.

RMSE: The Root Mean square error (RMSE), is given by [5]:

$$
\mathrm{RMSE}=\sqrt{\frac{1}{M N} \sum_{i=1}^{M} \sum_{j=1}^{N}\left(x_{i, j}-y_{i, j}\right)^{2}}
$$

PSNR: Peak Signal to Noise Ratio (PSNR) is computed using [10]:

$$
\text { PSNR }=20 \cdot \log _{10}\left(g^{2} \max / \mathrm{RMSE}\right)
$$

Where $g^{2} \max$ is the maximum intensity in the unfiltered images. The PSNR is higher for a better transformed image.

IMGQ: The universal Quality Index [14]:

$$
Q I=\frac{\sigma_{x y}}{\sigma_{x} \sigma_{y}} \frac{2 \bar{y} \bar{x}}{(\bar{y})^{2}+(\bar{x})^{2}} \frac{2 \sigma_{y} \sigma_{x}}{\sigma_{x}^{2}+\sigma_{y}^{2}}
$$

Where $\bar{x}$ and $\bar{y}$ represent the mean and $\sigma_{x}, \sigma_{y}$ the standard deviation of original and despeckled images. $\sigma_{x y}$ represents the covariance between the original and despeckled images.

SSIM: The Structural Similarity Index between two images is computed as [15]:

$$
S S I=\frac{(2 \bar{x} \bar{y}+2.55)\left(\sigma_{x y}+7.65\right)}{\left(\bar{x}^{2}+\bar{y}^{2}+2.55\right)\left(\sigma_{x}^{2}+\sigma_{y}^{2}+7.65\right)}
$$

The SSI lies between -1 for a bad and 1 for a good similarity between the original and despeckled images.

In ultrasound imaging in addition to speckle noise suppression, preservation of edges of the image also should be considered. Therefore in addition to the above performance metrics, another measure for edge preservation is also considered.

EPF: The edge preservation ability of the filter is compared by Edge Preservation Factor and is computed using [11]:

$$
\mathrm{EPF}=\frac{\sum(\Delta \mathrm{x}-\overline{\Delta \mathrm{x}})(\Delta \mathrm{y}-\overline{\Delta y})}{\sqrt{\sum(\Delta \mathrm{x}-\overline{\Delta \mathrm{x}})^{2} \sum(\Delta \mathrm{y}-\overline{\Delta y})^{2}}}
$$

Where $\Delta \mathrm{x}$ and $\Delta \mathrm{y}$ are the high pass filtered versions of images $\mathrm{x}$ and $y$, obtained with a $3 \times 3$ pixel standard approximation of the Laplacian operator. The larger value of EPF means more ability to preserve edges.

\section{RESULTS AND DISCUSSION}

The ultrasound image of liver is taken as a test image. Speckle noise of variance 0.02 has been added synthetically to the test image. To investigate the effectiveness of the different speckle reduction methods, they are applied to the image corrupted with speckle noise .Simulations are carried out in MATLAB.Table.1 presents the performance of different speckle reduction filters in terms of RMSE, SNR, PSNR, SSIM, IMGQ and EPF. The standard filters are tested with a window size of $3 \times 3$. To see the over-all performance, results are plotted in Fig. 1 and the qualitative results are shown in Fig.2. From Table 1, it can be seen that in the case of linear filters, the Lee and Kuan filters perform more or less equally and better than the mean and Frost filters. The standard median filter improves the signal strength when compared to homomorphic filter but has less edge preservation capability among the nonlinear filters compared. The NCD filter performs better than the median and homomorphic filters. The performance metrics show that amongst the different type of speckle reduction filters listed in Table.1, SRAD filter removes substantial amount of noise and also preserves edges and details.

\section{CONLUSION}

The speckle reduction and detail retention are two key issues in speckle suppression of medical ultrasound images. Performance of all algorithms is tested with ultrasound image of liver. Speckle Reducing Anisotropic Diffusion filter (SRAD) is better than several commonly used filters including Mean ,Lee, Kuan, Frost, median and also the homomorphic filter and Non linear Coherent Diffusion filter (NCD) in terms of speckle suppression and detail preservation. 
Table 1. Comparison of performance of different filters

\begin{tabular}{|l|l|l|l|l|l|l|}
\hline Filter & RMSE & SNR & PSNR & SSIM & IMGQ & EPF \\
\hline Mean & 6.535 & 20.377 & 34.387 & 0.8500 & 0.7123 & 0.2149 \\
\hline Lee & 6.259 & 21.186 & 35.211 & 0.8817 & 0.7439 & 0.2619 \\
\hline Kuan & 6.253 & 18.218 & 35.220 & 0.8798 & 0.7234 & 0.2630 \\
\hline Frost & 6.882 & 19.476 & 33.458 & 0.8134 & 0.7281 & 0.2432 \\
\hline Med & 7.523 & 20.801 & 34.837 & 0.8630 & 0.7182 & 0.3953 \\
\hline Homo & 5.209 & 18.247 & 33.706 & 0.8369 & 0.7241 & 0.6358 \\
\hline SRAD & 6.209 & $\mathbf{2 2 . 7 9 9}$ & $\mathbf{3 6 . 8 0 5}$ & $\mathbf{0 . 8 5 8 2}$ & 0.7067 & $\mathbf{0 . 7 2 6 3}$ \\
\hline NCD & 6.209 & 21.330 & 35.281 & 0.8468 & 0.9446 & 0.6232 \\
\hline
\end{tabular}
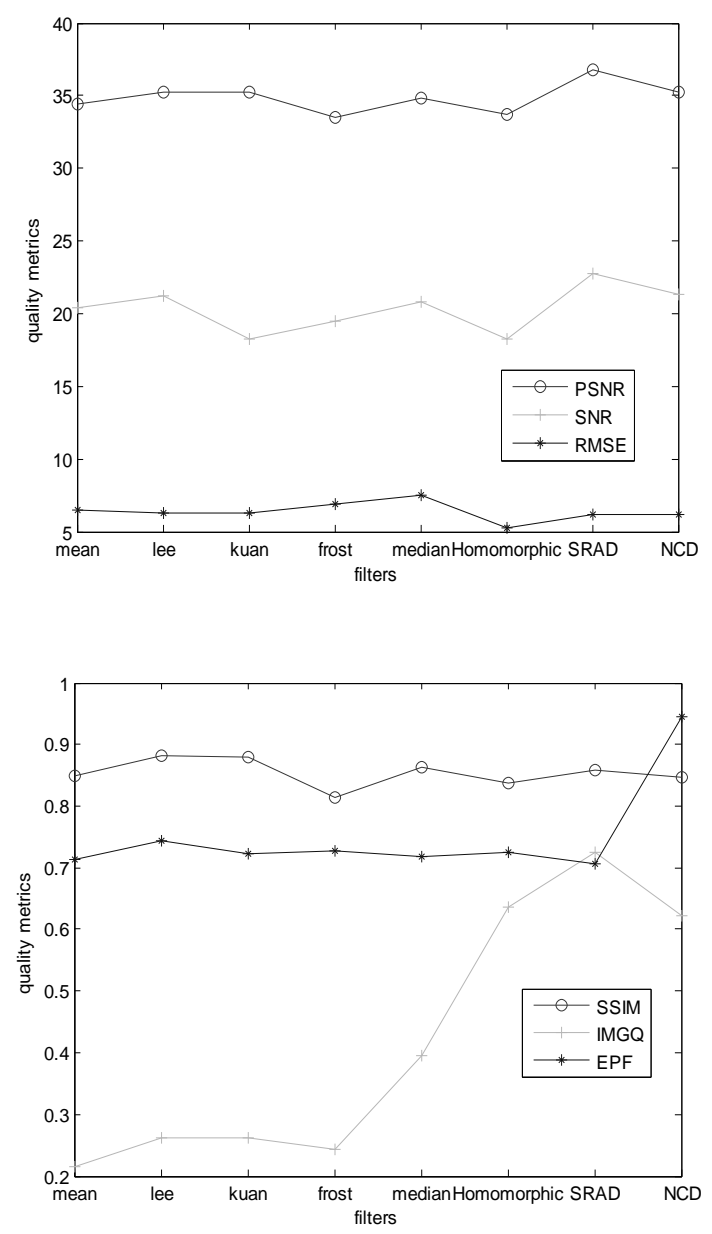

Fig.1.Filter's performance in terms of RMSE, SNR, PSNR, EPF, SSIM, IMGQ.
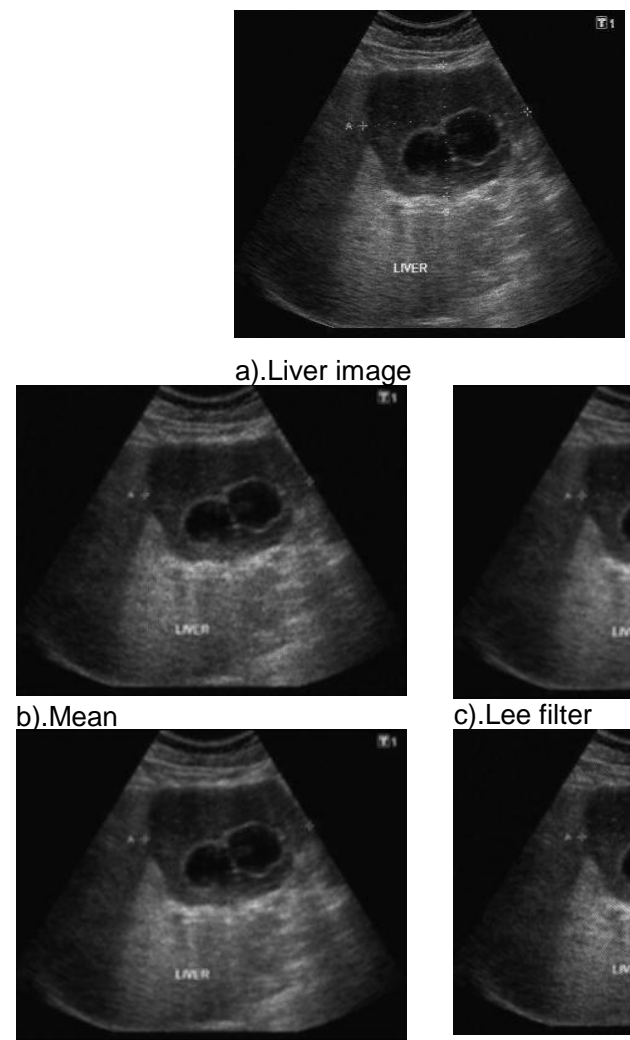

d).Kuan filter
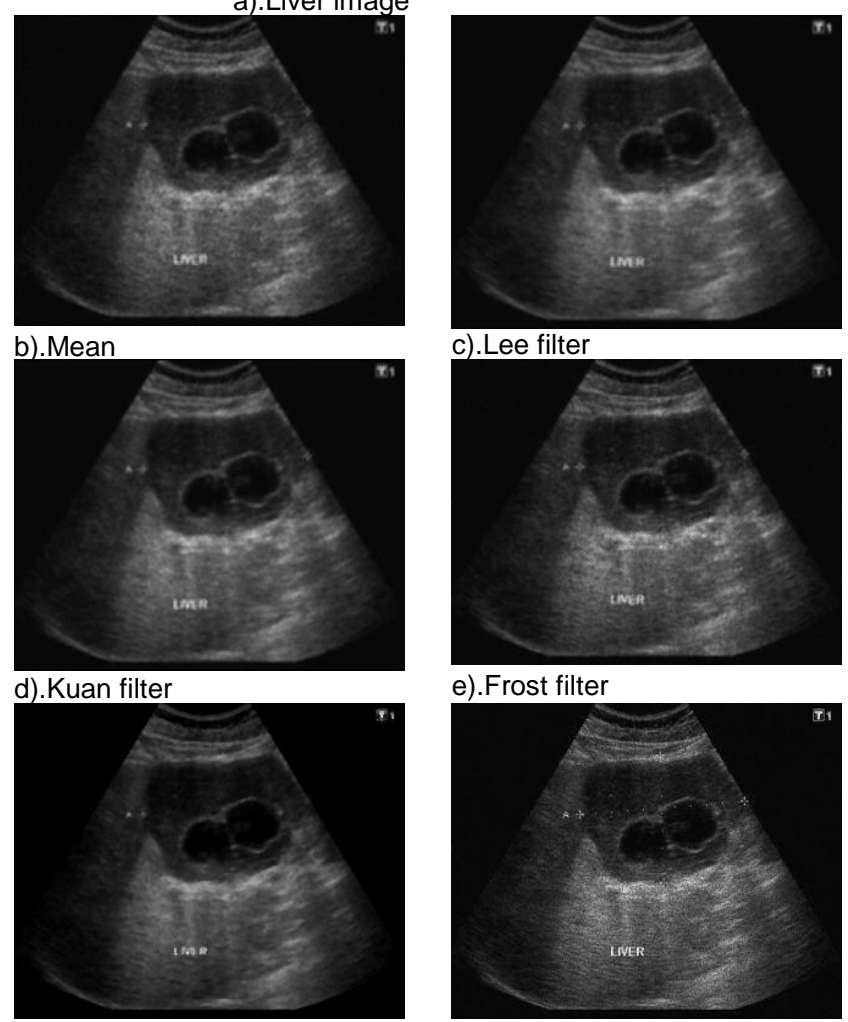

f).Median filter

g).Homomorphic

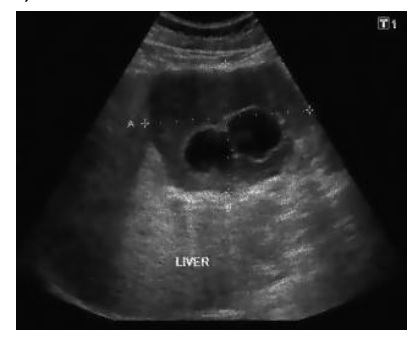

h)SRAD

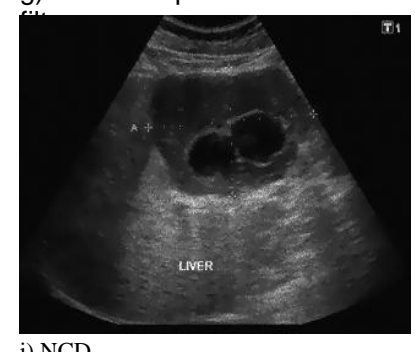

i).NCD

Fig. 2. Original ultrasound image of liver (a), and the filtered images (b)-(i).

\section{REFERENCES}

[1] J. G. Abbott and F. L. Thurnstone, "Acoustic speckle: Theory and experimental analysis," Ultrason. Imag., vol. 1, pp. 303324, 1979. 
[2] K. Z. Abd-Elmoniem, A. B. Youssef, and Y. M. Kadah, "Real-time speckle reduction and coherence enhancement in ultrasound imaging via nonlinear anisotropic diffusion," IEEE Trans. Biomed. Eng., vol.49, pp. 997-1014, 2002.

[3] C. B. Burckhardt, "Speckle in ultrasound B-mode scans," IEEE Trans.Sonic Ultrason., vol. SU-25, pp. 1-6, Jan. 1978.

[4] V.S.Frost,J.A.Stiles,K.S.Shanmugam and J.C.Holtzman, "A model for radar images and its application for adaptive digital filtering of multiplicative noise, "IEEE Transactions on pattern analysis and machine inelligence,Vol.4,No.2, pp.157- 165,1982.

[5] R.C. Gonzalez and R.E. Woods: 'Digital Image Processing', Addison- Wesley Publishing Company, 2002.

[6] A.K. Jain, Fundamental of Digital Image Processing. Englewood Cliffs, NJ: Prentice-Hall, 1989.

[7] D.T. Kuan, A.A. Sawchuk, T.C. Strand, and P. Chavel, "Adaptive restoration of images with speckle," IEEE Trans. ASSP., vol. 35,no. 3, pp. 373-383, March 1987.

[8] J.S. Lee, "Refined filtering of image noise using local statistics," Journal of Computer Graphic and Image Processing, vol. 15, pp. 380-389, 1981.

[9] Y. H. Lee and S. A. Kassam, "Generalized median filtering and related nonlinear filtering techniques." IEEE Trans. Acoust., Speech, Signal Processing, vol. ASSP-33, pp. 672 683, June 1985.
[10] D.Sakrison, "On the role of observer and a distortion measure in image transmission, "IEEE Transaction on Communication. Vol 25,pp. 1251-1267,November,1977.

[11] F.Sattar,L.Floreby ,G.Salomonsson, B.Lovstorm ,"Image enhancement based on a nonlinear multiscale method", Image processing IEEE transactions on,vol.6 ,no.6,pp.888$895,1997$.

[12] S.W. Smith and H. Lopez, "A contrast-detail analysis of diagnostic ultrasound imaging," Med. Phys., vol. 9, pp. 4-12, 1982.

[13] S.Solbo and T. Eltoft, "Homomorphic wavelet basedstatistical despeckling of SAR images," IEEE Trans. Geosc. Remote Sensing, vol. 42, no. 4, pp. 711-721, 2004.

[14] Z.Wang, A.Bovik, “A Universal Quality index,”IEEE Signal Processing Letters, vol.9, no.3, pp.81-84, March, 2002.

[15] Z.Wang, A.Bovik, H,Sheik and E.Simoncelli,"Image Quality assessment: From error measurement to structural similarity, "IEEE Transaction on imageprocessing,Vol.13,No.4,pp.600612,April,2004.

[16] Y. Yongjian and S. T. Acton, "Speckle reducing anisotropic diffusion,” IEEE Trans. Image Processing, vol. 11, no. 11, pp.1260-1270, Nov. 2002. 